\title{
A Novel High-resolution Dedicated Axillary PET Improve Sensitivity in the Assessment of Regional Nodal Spread of Disease in Early Breast Cancer.
}

\section{Jingyi Cheng}

Fudan University Shanghai Cancer Center

Junjie Li

Fudan University Shanghai Cancer Center

Guangyu Liu

Fudan University Shanghai Cancer Center

Ruohong Shui

Fudan University Shanghai Cancer Center

Sheng Chen

Fudan University Shanghai Cancer Center

Benlong Yang

Fudan University Shanghai Cancer Center

Shaoli Song

Fudan University Shanghai Cancer Center

Yingjian Zhang

Fudan University Shanghai Cancer Center

Zhimin Shao ( $\nabla$ zhimin_shao@yeah.net)

Fudan University Shanghai Cancer Center

\section{Research article}

Keywords: Lymph Node, Breast Cancer, Molecular Imaging, New PET technology

Posted Date: September 3rd, 2020

DOI: https://doi.org/10.21203/rs.3.rs-69371/v1

License: (c) (i) This work is licensed under a Creative Commons Attribution 4.0 International License.

Read Full License 


\section{Abstract}

Background: The aim of this study was to assess if a novel high-resolution dedicated axillary lymph node Positron Emission Tomography (LymphPET) system could improve sensitivity analysing Lymph Node involvement in early breast carcinoma with clinical N0-N1 stage.

Methods: A total of 103 patients with clinical stage of T1-2N0-1M0 breast cancer were evaluated with LymphPET. The maximum single-voxel PET uptake value of Axillary Lymph Node (maxLUV) and the tumor-to-background ratio (TBR) for fat (TBR1) and muscle (TBR2) tissue were calculated. Then, seventyeight patients with cN0 stage received sentinel lymph node biopsy (SLNB) alone or in combination with ALN dissection (ALND) and 25 patients with cN1 stage underwent fine-needle aspiration (FNA).

Results: Ninety-nine invasive breast carcinoma entered this study. The diagnostic sensitivity of LymphPET was $87.80 \%$, specificity was $79.31 \%$, false-negative rate was $12.20 \%$, false-positive rate was $20.69 \%$, positive predictive value was $75.00 \%$, negative predictive value was $90.20 \%$, and accuracy was $82.83 \%$. The maxLUV was superior to TBR1 and TBR2 in the detection of ALN, with 0.27 being the most optimal cutoff value.

Conclusion: The ${ }^{18}$ F-FDG LymphPET could be used to identify and recognize more indolent ALNs of the breast cancer because of more sensitivity and much higher negative predictive value.

\section{Introduction}

Over the last 2 decades, with the improving systemic therapies and improved molecular tools, breast surgery has move from surgical axillary lymph node dissection (ALND) to the much less-radical surgical sentinel lymph node excision biopsy (SLNB), the current reference standard. Now, there is a global paradigm shift toward de-escalation of axillary lymph node (ALN), called "less is more". The consensus base on recent evidence suggesting lack of benefit from overaggressive treatment [1-3]. In order to avoid overtreatment, a non-invasive method with more sensitivity and much higher negative predictive values $\triangle N P V \bigotimes$ needed to identify and recognize more indolent ALNs of the breast cancer.

${ }^{18} \mathrm{~F}$-fluorodeoxyglucose $\left({ }^{18} \mathrm{~F}\right.$-FDG) whole-body positron emission tomography/computed tomography (WB-PET/CT) has been widely used in assessing the response to neoadjuvant treatment of primary tumors [4-5] and for identifying distant metastases. However, this technique is not ideal in the evaluation of ALN status in early breast cancer. This is mainly due to the fact that current WB-PET/CT systems typically yield reconstructed images with a resolution of 5-15 $\mathrm{mm}$ depending on the injected dose, imaging time, post-reconstruction filtering, as well as the intrinsic resolution of the scanner. This reduces their utility in detecting small lesions $(<1 \mathrm{~cm})$ and/or those with low tracer uptake [6]. As the result, although some authors had found detectability rates as high as $92 \%$ with WB PET/CT, these were achieved with tumours larger than $2 \mathrm{~cm}$ [7]. 
Newer imaging techniques offers exciting potential to assess the lower-risk and small size of ALN more sensitively. Dedicated axillary lymph node PET (LymphPET) is a new technological development of PEMTECH Co., Ltd. Not only is the spatial resolution of LymphPET much higher than that of WB-PET/CT, it is also more sensitive in the detection of small, hot ${ }^{18} \mathrm{~F}-\mathrm{FDG}$-avid lesions, such as subclinical ALN.

The aim of this study was to assess if LymphPET could improve sensitivity in early breast carcinoma with clinical NO-N1 stage and if the NPV would be high enough to recognize the more indolent ALNs noninvasively.

\section{Materials And Methods}

This was a retrospective, single-center study including patients whose primary breast cancer was confirmed to be malignant by core needle biopsies. ${ }^{18} \mathrm{~F}$-FDG LymphPET was performed before ALN assessment (ALNA). The LymphPET results were compared with the classical "gold standard" pathological assessment of ALN: SLNB alone or in combination with ALND for resectable tumor, and fineneedle aspiration (FNA) and neoadjuvant treatment for unresectable tumor.

\section{Patients}

Patients aged between 18 and 70 years with clinical T1-2N0-1M0 (cT1-2N0-1M0) breast cancer were enrolled from September to December. Tumor staging was based on the eighth edition of the American Joint Committee on Cancer staging manual. Compared with pathological $\mathrm{N}$ category $(\mathrm{pN})$, which based on microscopic assessment of at least one node required, include imaging and diagnostic biopsy, the clinical $\mathrm{N}$ category $(\mathrm{cN})$ based on physical exam, imaging, FNA or core needle biopsy, sentinel node biopsy. The cN0 means no regional lymph metastases (by imaging or clinical examination). The cN1 means metastases to movable ipsilateral level II axillary lymph node(s)[8]. In our study, both the primary and ALN status were evaluated by ultrasound (US). For CNO patients, ALN was pathologically assessed using SLNB alone or SLNB and ALND. For CN1 ALN was pathologically confirmed using FNA.

The exclusion criteria including: uncontrolled diabetes, pregnant or breastfeeding, had already undergone neoadjuvant treatment, SLNB, or ALND, or had any clinical evidence of distant metastases at the time of enrolment. The study was approved by the Institutional Ethics Committee and signed informed consent was obtained from every patient prior to participation in this study.

\section{LymphPET System and Examination}

The LymphPET (PEMTECH ${ }^{\mathrm{TM}}$, Shanghai, China) device contains movable double-planar confronted detectors with an axilla capability view and an adjustable distance between the two detector plates from 10 to $30 \mathrm{~cm}$. Size of the sensitive detection area is $208 \times 208 \mathrm{~mm}$ with a useful field of vision (FOV) of $204 \times 204 \mathrm{~mm}$, consisting of 16 modules in each plate with LYSO crystals of $1.2 \times 1.2 \times 12 \mathrm{~mm}$, and coupled to a new Double Readout Module (DRM) system with J6 series SiPM arrays. The results show a 
transaxial resolution of $1.4 \mathrm{~mm}$, uniformity of $10 \%$, timing resolution of $500 \mathrm{ps}$, true coincidence rate peak of 390 kcps @20 MBq, and NEC rate peak of 319 kcps @12.6 MBq.

Patients were fasted at least $6 \mathrm{~h}$ prior to receiving a standardized injection of $4 \mathrm{mCi}{ }^{18} \mathrm{~F}-\mathrm{FDG}$. Blood glucose levels were required to be lower than $10 \mathrm{mmol} / \mathrm{L}$. After a resting period of $60 \mathrm{~min}$ to allow tracer distribution, LymphPET was performed with the patient standing up and abduction arm (Fig. 1).

\section{LymphPET Image Analysis}

In order to postulate a precise method of quantification adapted to this geometry, the concept of LUV, as a surrogate for SUV, or LymphPET Uptake Value, has been used for this special biplanar symmetry. The LUV, like SUV characterizes the activity intensity in the image, but unlike the SUV, the quantification of LUV does not suffer the same data corrections such as attenuation or scattered photons [9]. Although validity of the LUV as a quantification metric has not yet been demonstrated due to its novelty, a similar concept is generally accepted [10-12] PUV (PEM Uptake Value) being demonstrated in a large single study published in the AJR ${ }^{11}$ a statistically significant correlation between PUV and SUV in the breast.

Image evaluation and quantification of maximum single-voxel LymphPET Uptake Value (maxLUV) was performed using commercial Medical Image Merge (version 6.5.4; MIM Software Inc, OH, USA), which is the professional image processing software certified by the United States Food and Drug Administration. Two separated experienced nuclear medicine physicians with 10 years of experience in PET / CT, who were blinded to study-related information apart from the laterality of breast cancer, analyzed the images and marked the regions of interest (ROIs) manually.

ALNs were considered positive under the following three conditions: (1) the positive focus was located in the axillary region but not in skin, muscle, or bone; (2) ${ }^{18} \mathrm{~F}-\mathrm{FDG}$ uptake was higher than the reference background (fat tissue); and (3) the physiological lymphatic uptake was excluded. For quantitative analysis, the size of the lymph node ( $\mathrm{LN})$ was measured and an elliptic-shaped region of interest (ROI) was drawn manually. ${ }^{18} \mathrm{~F}-\mathrm{FDG}$ uptake into this ROI was calculated as ALN maxLUV (maxLUV). The highest maxLUV was selected as study value in case multiple LNs were detected. Additionally, three separate ROls measuring $1 \mathrm{~cm}$ in diameter (fat background) were located the axillary adipose tissue, and the mean value of those areas was defined as maxLUV fat. Moreover, three 1-cm diameter ROls were located at the biceps brachii and ectopectoralis muscles (muscle background) and the mean value was

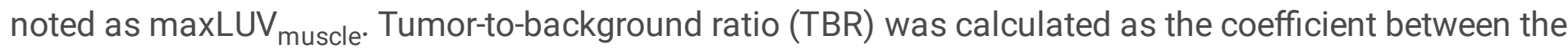
positive value of maxLUV and the corresponding background value: TBR1=maxLUV/maxLUV fati TBR2=maxLUV/maxLUV $V_{\text {muscle. }}$ Thus, three parameters were calculated for each patient: maxLUV, TBR1, and TBR2.

\section{Surgery and Pathological Evaluation}


For cN0 patients, ALN was pathologically assessed using SLNB alone or SLNB and ALND. For CN1, ALN was pathologically confirmed using FNA. If FNA was negative, SLNB was performed. If FNA was positive, neoadjuvant treatment was performed. For those patients with no more than two SLN positives, the decision whether to perform further axillary dissection depended on the operative type (breast-conserving therapy or mastectomy) and individual pathological characteristics. All LymphPET scans were performed before FNA.

In SLNB, nodes were identified by injection of blue dye (methylthioninium chloride, Jiangsu Jumpcan Pharmaceutical Co., Ltd.) and/or by intraoperative guidance of the gamma detector probe (neo2000,

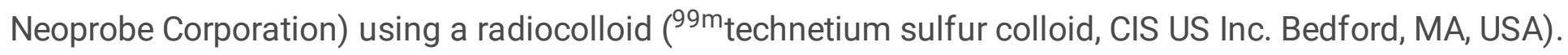
In our study, SLNs were defined as any blue-stained node, any node with a blue-stained lymphatic channel directly leading to it, any node with radioactive counts $10 \%$ or more of the most radioactive node, or any pathologically palpable nodes. Internal mammary SLNB would not be performed even if the nodes were detected using lymphoscintigraphy.

Touch imprint cytology was routinely performed on every SLN that was harvested. SLNs were cut along the long axis at a 2.0- to 3.0-mm interval intraoperatively, and each cut surface was touched, at least three times, onto a clean glass slide and stained with hematoxylin and eosin. Staining of serially-sectioned slices was performed in 100- $\mu$ m intervals on every node. Slides were sent for cytopathological examination immediately after preparation. Moreover, additional slices were formalin-fixed and paraffinembedded for further evaluation. According to pathological results, ALNs were classified as macrometastasis $(>2.0 \mathrm{~mm})$, micro-metastasis $(0.2-2.0 \mathrm{~mm})$ and isolated tumor cells $($ ITC, $<0.2 \mathrm{~mm})$ according to the tumor-node-metastasis staging system.

It should be noted that both ITC and micro-metastases $(<2 \mathrm{~mm})$ were considered negative in the final statistical analysis.

\section{Statistical Analysis}

Data were presented as range with means and standard deviation. Receiver operating characteristic (ROC) curves and area under the ROC curves (AUCs) were employed to evaluate the predictive value of ${ }^{18}$ F-FDG LymphPET, by determining the optimum cutoff for the three recorded parameters. Logistic regression analysis was used to determine independent factors predictive of ALN metastases. Multiple linear regression analysis was used to confirm whether maxLUV correlated with different clinical factors. For categorical variables, differences were calculated using the Chi-squared test or Fisher's exact test where applicable. Statistical analysis was performed using the SPSS 20.0 software (IBM Inc., Chicago, IL, USA), and two-sided $p$ values were reported with an alpha of $0.05(p<0.05)$.

\section{Results}

Between September and December 2019, 103 cases were registered. Four cases were excluded because the primary lesions were confirmed to be TIS (Tumor In situ, Carcinoma in situ, or stage 0 cancer). Finally, 
99 cases with invasive carcinoma were included, the clinical and anatomopathological characteristics of which are shown in Table 1. Of these, 41 (41.41\%) were ALN metastatic cases, of which 16 cases were confirmed by SLNB alone or SLNB with ALND, and 25 by FNA (Fig. 2).

\section{Diagnostic Accuracy of LymphPET}

The diagnostic sensitivity (87.80\%), specificity $(79.31 \%)$, false-negative rate $(12.20 \%)$, false-positive rate (20.69\%), positive predictive value (PPV, 75.00\%), negative predictive value (NPV, 90.20\%), and accuracy (82.83\%) of LymphPET are summarized in Fig. 3, and a clear example of a truly positive case and its assessment with other imaging techniques are visualized in Fig. 4. All five false-negative cases were of Luminal subtype with nuclear grade II. Two of them were negative and three displayed enlarged nodes, but no metastasis, in axillary US. Of these 5 patients, 3 to 5 SLNs were biopsied, and we found 1 macrometastasis in 3 patients each, 2 macro-metastases in 1 patient and 1 patient conformed by FNA. Then, 4 patients with maxLUV of " 0 " underwent ALND with 0/16, 1/16, 3/16 and 3/19 in finally ALND surgery and 1 patient with maxLUV of 0.16 underwent NAC. Twelve false-positives (FPs) were identified, including 7 Luminal subtypes, and 5 triple-negative breast cancer (TNBC) subtypes, with 4 nuclear grade lls and 8 nuclear grade IIIs. In 2 of 12 patients, axillary US was negative, whereas the remaining 10 patients displayed enlarged LNs measuring up to $20 \times 30 \mathrm{~mm}$. To understand these type of results, examples of true-positive and false-positive cases are shown in Fig. 5.

\section{Comparison Between maxLUV, TBR1, and TBR2}

Forty-eight patients had positive LymphPET scans in ALNs, with a range in maxLUV from 0.16 to 5.05 $(1.13 \pm 1.11)$. The maxLUV fat background ranged from 0.02 to $0.15(0.07 \pm 0.02)$ and the maxLUV muscle $_{\text {f }}$ background ranged from 0.13 to $0.33(0.23 \pm 0.05)$. The TBR1 ranged from 2.66 to $55.75(14.11 \pm 11.88)$ and TBR2 ranged from 0.94 to 16.93 (4.42 \pm 3.65$)$. Strong correlations were found between maxLUV and TBR1 $(r=0.81, p<0.05)$, and maxLUV and TBR2 $(r=0.97, p<0.05)$. We identified 48 positive nodes including 36 true-positive (TP) nodes and 12 FP nodes (Table 2 ). There were statistically significant differences exist in maxLUV, TBR1 and TBR2 of TP and FP groups but not exist in size. These results indicate that maxLUV, TBR1, and TBR2, but not size, could be used to distinguish TP from FP LNs.

ROC curves of the three selected parameters for predicting nodal metastasis are shown in Fig. 6. AUC values for maxLUV, TBR1, and TBR2 were 0.895 (95\% Cl, 0.828-0.962), 0.885 (95\% Cl, 0.815-0.954) and $0.884(95 \% \mathrm{Cl}, 0.814-0.954)$, respectively. The diagnostic yield of TBR1 and TBR2 was inferior to that of maxLUV. Therefore, maxLUV was used in the following analysis.

According to the ROC curve and most optimal Youden's Index, when the maxLUV cutoff value was set at 0.27 , the most optimal sensitivity and specificity were $87.80 \%$ and $79.31 \%$, respectively. On the other hand, we noted that if the maxLUV cutoff value was set at 0.69 , the specificity was $98.30 \%$ but the sensitivity was only $51.20 \%$.

\section{Correlation Between maxLUV and Other Parameters}


In the multivariate analysis, primary tumor size, nuclear grade, ER positive, HER2 high expression, and the proliferation marker Ki67 were not independent predictive factors of LN metastasis ( $p>0.05$ for all). Using logistic regression, we found that maxLUV of LNs was affected by the size of the primary tumor and by Ki67 expression, indicating that the larger the tumor or the higher Ki67 levels, the greater the chance that LymphPET correctly detected the absence of nodal disease. However, it should be noted that for positive tests, the obtained results were inconclusive due to $20.69 \%$ false-positive rate.

\section{Discussion}

There is a global paradigm shift toward de-escalation of axillary management on the basis of recent evidence suggesting lack of benefit from overaggressive treatment [13]. Hence, it is important to distinguish between the patient who is clinically node-positive and who is clinically node-negative but has a positive SLNs. Presently, SLNB is standard initial workup in breast cancer patients who are clinically node-negative with about $9.8 \%$ of false-negative rate [14]. If there is a non-invasive molecular tool could achieve the same false-negative rate, it will validate the non-inferiority with SLNB in future axillary management.

During the last year, different companies and projects have been working on this type of open dedicatedPET systems. Even we have been testing another prototype with a similar geometry [15] but with very different characteristics, both in detectors (simple versus double reading modules), crystals (continuous versus pixelated), mechanics (manual versus automatic), the TOF capability (absent versus present) and the software (external versus adhoc). Finally, due to its technical specifications, its versatility and simplicity of use, as well as the highest resolution and quantification capacity, the system we have selected to go through clinical validation and future studies in patients has been the LymphPET (PEMTECH ${ }^{\mathrm{TM}}$ ).

In the present study, LymphPET increased diagnostic sensitivity greatly to $87.80 \%$, thereby reducing falsenegative rate (12.20\%) and enhancing NPV (90.20\%). Of all 99 cases, 41 were pathologically confirmed to be macro-metastasis. LymphPET was accurate in the diagnosis of 36 cases while leading to misdiagnosis in 5 cases, and the NPV (90.20\%) was much higher than that of WB-PET/CT. Further analysis revealed that these five cases of FNs were all of Luminal subtype with nuclear grade II, suggesting that lower proliferation and metabolism leads to reduced FDG uptake. Additionally, ITCs ( $<0.2$ $\mathrm{mm})$ and micro-metastases $(0.2-2 \mathrm{~mm})$ were defined as pathologically negative because they are too difficult to detect by any imaging technique, and several recent studies have shown that micrometastases do not influence overall survival [16-18].

Compared to other current non-invasive diagnostic modalities of ALNs include US, mammography (MMG), magnetic resonance imaging (MRI) and WB-PET/CT. While US remains one of the key tools, it bears the limitation of being subjective [19]. A systematic review analyzing the use of US has shown significant variation between institutions, with overall sensitivity ranging between $26 \%-76 \%$ and a specificity between $88 \%-98 \%$ [20]. A more recent meta-analysis involving 21 studies found that US 
assessment of abnormal nodes allowed a median sensitivity of $64 \%$ and a specificity of $82 \%$ [21]. MMG is more suitable for the examination of breast disease but is not considered reliable for ALN evaluation, because part of the axillary area may not be visualized by routine MMG [22]. MRI is mostly used for assessment of newly diagnosed breast cancer and to examine the response to neoadjuvant treatment, but is also limited by an insufficient imaging field of the axillary region [23]. While WB-PET/CT is recognized to be sufficiently sensitive for the detection of distant metastases, it is not suitable to rule out ALN involvement. Wahl et al. [24] compared PET/CT results with pathological findings in a prospective multi-center trial including 360 cases of newly diagnosed invasive breast cancer. They found $61 \%$ sensitivity and $80 \%$ specificity for axillary metastases, with a PPV of $62 \%$ and NPV of $79 \%$.

Another aim of the study is to identify the most suitable indicator in the detection of ALN using LymphPET. Therefore, in addition a new concept, as a surrogate for SUV, LUV, two TBRs were used with adipose and muscle tissue in the axillary region selected as background. Our results demonstrate that while the three parameters were highly correlated, maxLUV showed the highest AUC, revealing that it was still the most reliable indicator, also in LymphPET. When the cutoff value of maxLUV $\geq 0.27$ recommended by Youden's Index is confirmed, the sensitivity of diagnose is $87.80 \%$ and the NPV reach a maximum of $90.20 \%$, which is about the same level of NPV of intraoperative cytology (88.1\%) [14]. When the cutoff value of maxLUV $\geq 0.69$ is chosen, a maximum of specificity $(98.30 \%)$ is reached.

Consequently, the cutoff value of maxLUV should be chosen depending on the clinical context: if a maximum of NPV is desired, 0.27 is more suitable, whereas a high specificity for confirmation of LN metastasis can be better achieved using 0.69 .

Interestingly, using logistic regression analysis, we observed that both the size of the primary lesion as well as Ki67 levels influence maxLUV, whereby the size of the primary tumor, as well as proliferative activity indicated by Ki67 expression, positively correlated with ALN metastasis. These findings are consistent with previous studies [25] and indicate that LymphPET produces a higher NPV in patients with larger primary lesions, higher Ki67 levels, HER2 subtypes, or TNBC.

The limitations of our study are the absence of WB PET imaging as the comparation of same patient. Another limitation is the comparably small number of patients. A future, prospective study (NCT04072653, SOAPET trial) aimed at applying of LymphPET to ALN management is ongoing.

\section{Conclusion}

The ${ }^{18}$ F-FDG LymphPET could be used to identify and recognize more indolent ALNs of the breast cancer because of and much higher sensitivity and lower false-negative rate. The newer imaging techniques raises the possibility that in the future such techniques may obviate need for surgical SLNB in select patient populations.

\section{Declarations}


Ethics approval and consent to participate: The study was approved by the Institutional Ethics Committee. Signed informed consent was obtained from each patient.

Consent for publication: Not applicable

Availability of data and materials: The datasets used and/or analysed during the current study are available from the corresponding author on reasonable request.

Competing interests: The authors declare that they have no competing interest.

Funding: Not applicable

\section{Authors' contributions:}

- JC: conceived of the presented idea, performed the analysis, drafted the manuscript and designed the figures, carried out the experiment, wrote the manuscript.

- JL: conceived of the presented idea, carried out the experiment, wrote the manuscript, verified the numerical results.

- GL: carried out the experiments, verified the numerical results.

- RS: verified the analytical methods, processed the experimental data.

- SC: carried out the experiment, contributed to the interpretation of the results.

- BY: verified the analytical methods, processed the experimental data.

- SS: carried out the experiments, contributed to the interpretation of the results.

- YZ: supervised the findings of this work, supervised the Project, contributed to the interpretation of the results.

- ZS: conceived of the presented idea, supervised the findings of this work, supervised the project.

All authors read and approved the final manuscript

Acknowledgements: Not applicable.

\section{References}

1. Laura Esserman , Etienne Gallant, Michael Alvarado. Less Is More: The Evolving Surgical Approach to Breast Cancer. Am Soc Clin Oncol Educ Book. 2016;35:e5-e10.

2. Riegger C, Herrmann J, Nagarajah J, et al. Whole-body FDG PET/CT is more accurate than conventional imaging for staging primary breast cancer patients. Eur J Nucl Med Mol Imaging. 2012;39:852-863.

3. Koolen BB, Valdés Olmos RA, Elkhuizen PHM, et al. Locoregional lymph node involvement on 18FFDG PET/CT in breast cancer patients scheduled for neoadjuvant chemotherapy. Breast Cancer Res Treat. 2012;135:231-240. 
4. Wu S, Wang Y, Li J, et al. Subtype-Guided (18)F-FDG PET/CT in Tailoring Axillary Surgery Among Patients with Node-Positive Breast Cancer Treated with Neoadjuvant Chemotherapy: A Feasibility Study. Oncologist. 2019:theoncologist.2019-0583.

5. Cheng J, Wang Y, Mo M, et al. 18F-fluorodeoxyglucose (FDG) PET/CT after two cycles of neoadjuvant therapy may predict response in HER2-negative, but not in HER2-positive breast cancer. Oncotarget. 2015;6:29388-29395.

6. Koolen BB, Aukema TS, González Martínez AJ, et al. First clinical experience with a dedicated PET for hanging breast molecular imaging. Q J Nucl Med 2013;57:92-100.

7. Mahner S, Schirrmacher S, Brenner W, et al. Comparison between positron emission tomography using 2-[fluorine-18]fluoro-2-deoxy-D-glucose, conventional imaging and computed tomography for staging of breast cancer. Ann Oncol. 2008;19:1249-1254.

8. Giuliano AE, Edge SB, Hortobagyi GN. Eighth Edition of the AJCC Cancer Staging Manual: Breast Cancer. Ann Surg Oncol. 2018;25:1783-1785.

9. Rahim, S., Mawlawi, O., Fox, P., Taylor, S., Millican, R., Swanston, N. M., ... \& Rohren, E. M. (2014). Correlation of LUV and LUV in the extremities while using PEM as a high-resolution positron emission scanner. Skeletal radiology, 43(4), 453-458.

10. MacDonald $L$, et al. Clinical imaging characteristics of the positron emission mammography camera: PEM Flex Solo II. J Nucl Med. 2009;50(10):1666-75.

11. Wang $\mathrm{CL}$, et al. Positron emission mammography: correlation of estrogen receptor, progesterone receptor, and human epidermal growth factor receptor 2 status and 18F-FDG. AJR Am J Roentgenol. 2011;197(2):W247-55.

12. Eo JS, et al. Imaging sensitivity of dedicated positron emission mammography in relation to tumor size. Breast. 2012;21(1):66-71.

13. Yan M, Abdi MA, Falkson C. Axillary Management in Breast Cancer Patients: A Comprehensive Review of the Key Trials. Clin Breast Cancer. 2018;18:e1251-e1259.

14. Krag DN, Anderson SJ, Julian TB, et al. Technical outcomes of sentinel-lymph-node resection and conventional axillary-lymph-node dissection in patients with clinically node-negative breast cancer: results from the NSABP B-32 randomised phase III trial. Lancet Oncol. 2007 Oct;8(10):881-888.

15. Moliner, L., Zhang, C., Alamo, J., Morera, C., Jiménez-Serrano, S., Cuallado, J., ... \& Benlloch, J. M. (2020, May). Biplanar breast PET: preliminary evaluation. In 15th International Workshop on Breast Imaging (IWBI2020) (Vol. 11513, p. 115131R). International Society for Optics and Photonics.

16. Donker M, van Tienhoven G, Straver ME, et al. Radiotherapy or surgery of the axilla after a positive sentinel node in breast cancer (EORTC 10981-22023 AMAROS): a randomised, multicentre, openlabel, phase 3 non-inferiority trial. Lancet Oncol. 2014;15:1303-1310.

17. Giuliano AE, Hunt KK, Ballman KV, et al. Axillary dissection vs no axillary dissection in women with invasive breast cancer and sentinel node metastasis: a randomized clinical trial. JAMA. 2011;305:569-575. 
18. Giuliano AE, Ballman KV, McCall L, et al. Effect of Axillary Dissection vs No Axillary Dissection on 10Year Overall Survival Among Women With Invasive Breast Cancer and Sentinel Node Metastasis: The ACOSOG Z0011 (Alliance) Randomized Clinical Trial. JAMA. 2017;318:918-926.

19. Lee B, Lim AK, Krell J, et al. The efficacy of axillary ultrasound in the detection of nodal metastasis in breast cancer. AJR Am J Roentgenol. 2013;200:W314-W320.

20. Alvarez S, Añorbe E, Alcorta P, López F, Alonso I, Cortés J. Role of sonography in the diagnosis of axillary lymph node metastases in breast cancer: a systematic review. AJR Am J Roentgenol. 2006;186:1342-1348.

21. Houssami N, Ciatto S, Turner RM, Cody HS, 3rd, Macaskill P. Preoperative ultrasound-guided needle biopsy of axillary nodes in invasive breast cancer: meta-analysis of its accuracy and utility in staging the axilla. Ann Surg. 2011;254:243-251.

22. Shetty MK, Carpenter WS. Sonographic evaluation of isolated abnormal axillary lymph nodes identified on mammograms. J Ultrasound Med. 2004;23:63-71.

23. van Nijnatten TJA, Ploumen EH, Schipper RJ, et al. Routine use of standard breast MRI compared to axillary ultrasound for differentiating between no, limited and advanced axillary nodal disease in newly diagnosed breast cancer patients. Eur J Radiol. 2016;85:2288-2294.

24. Wahl RL, Siegel BA, Coleman RE, Gatsonis CG, Group PETS. Prospective multicenter study of axillary nodal staging by positron emission tomography in breast cancer: a report of the staging breast cancer with PET Study Group. J Clin Oncol. 2004;22:277-285.

25. Kanyılmaz G, Yavuz BB, Aktan M, Karaağaç M, Uyar M, Fındık S. Prognostic Importance of Ki-67 in Breast Cancer and Its Relationship with Other Prognostic Factors. Eur J Breast Health. 2019;15:256261.

\section{Tables}

Table 1. Anatomopathological characteristics of patients $(n=99)$ 


\begin{tabular}{|c|c|c|}
\hline Characteristics & No & Range/ Mean \\
\hline Age (years) & & $19-67 / 47.41$ \\
\hline Weight (kg) & & $43-77 / 58.96$ \\
\hline Breast tumor size (mm) & & $7-48 / 23.03$ \\
\hline Characteristics & No & $\%$ \\
\hline \multicolumn{3}{|l|}{ Tumor grade } \\
\hline ॥ & 40 & 57.97 \\
\hline III & 29 & 42.03 \\
\hline \multicolumn{3}{|l|}{ T category } \\
\hline T1 & 35 & 36.45 \\
\hline T2 & 61 & 63.55 \\
\hline Local resection & 3 & \\
\hline \multicolumn{3}{|l|}{$\mathrm{N}$ category } \\
\hline No & 74 & 74.75 \\
\hline N1 & 25 & 25.25 \\
\hline \multicolumn{3}{|l|}{ Subtype } \\
\hline Luminal & 66 & 66.67 \\
\hline HER2 & 10 & 10.10 \\
\hline TNBC & 23 & 23.23 \\
\hline \multicolumn{3}{|l|}{ Treatment } \\
\hline Mastectomy & 74 & 74.75 \\
\hline NAT & 25 & 25.25 \\
\hline
\end{tabular}

Table 2 Difference of Parameters in TP and FP groups $(n=48)$ 


\begin{tabular}{|lllll|}
\hline Parameter & TP $(\mathbf{n}=36)$ & FP $(\mathbf{n}=12)$ & $t$ & $P$ \\
\hline LN size $(\mathrm{mm})$ range & $4-25$ & $4-15.5$ & & \\
\hline LN size $(\mathrm{mm})$ mean & $10.49 \pm 5.35$ & $7.35 \pm 3.44$ & -1.965 & $0.39>0.05$ \\
\hline maxSUV In range & $0.16-4.9$ & $0.2-0.7$ & & \\
\hline maxSUV In mean & $1.34 \pm 1.13$ & $0.46 \pm 0.15$ & -2.778 & $0.001<0.05$ \\
\hline TBR1 range & $2.67-78.75$ & $4-13$ & & \\
\hline TBR1 mean & $17.13 \pm 15.42$ & $7.67 \pm 3.23$ & -2.182 & $0.02<0.05$ \\
\hline TBR2 range & $0.94-16.93$ & $1.25-5.20$ & & \\
\hline TBR2 mean & $5.13 \pm 3.98$ & $2.40 \pm 1.02$ & -2.432 & $0.001<0.05$ \\
\hline
\end{tabular}

LN size =axillary lymph node size measured by X-PET

TP =true positive; $F P=$ false positive; $T B R=$ tumor background ratio

\section{Figures}

Figure 1.
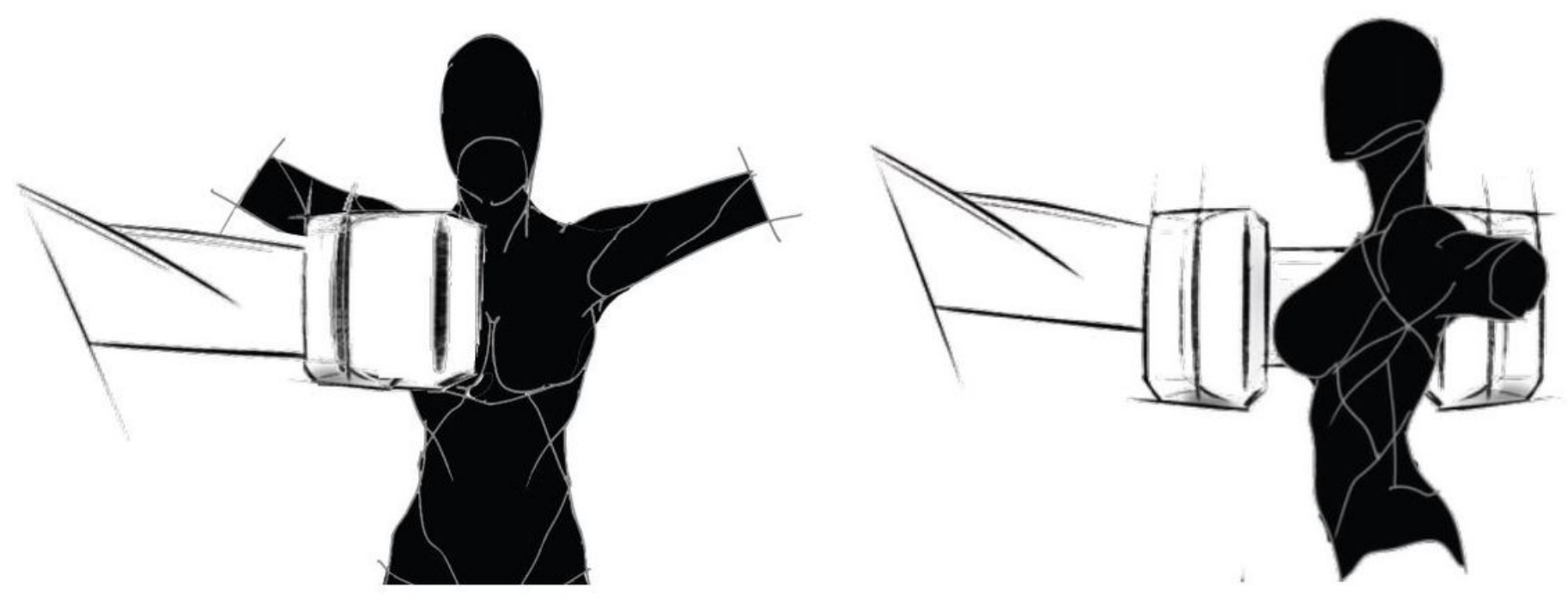

Figure 1 
Diagram of dedicated axillary lymph node positron emission tomography (LymphPET) geometry and patient's position: frontal (left) and lateral view (right).

Figure 2.

Lymph PET

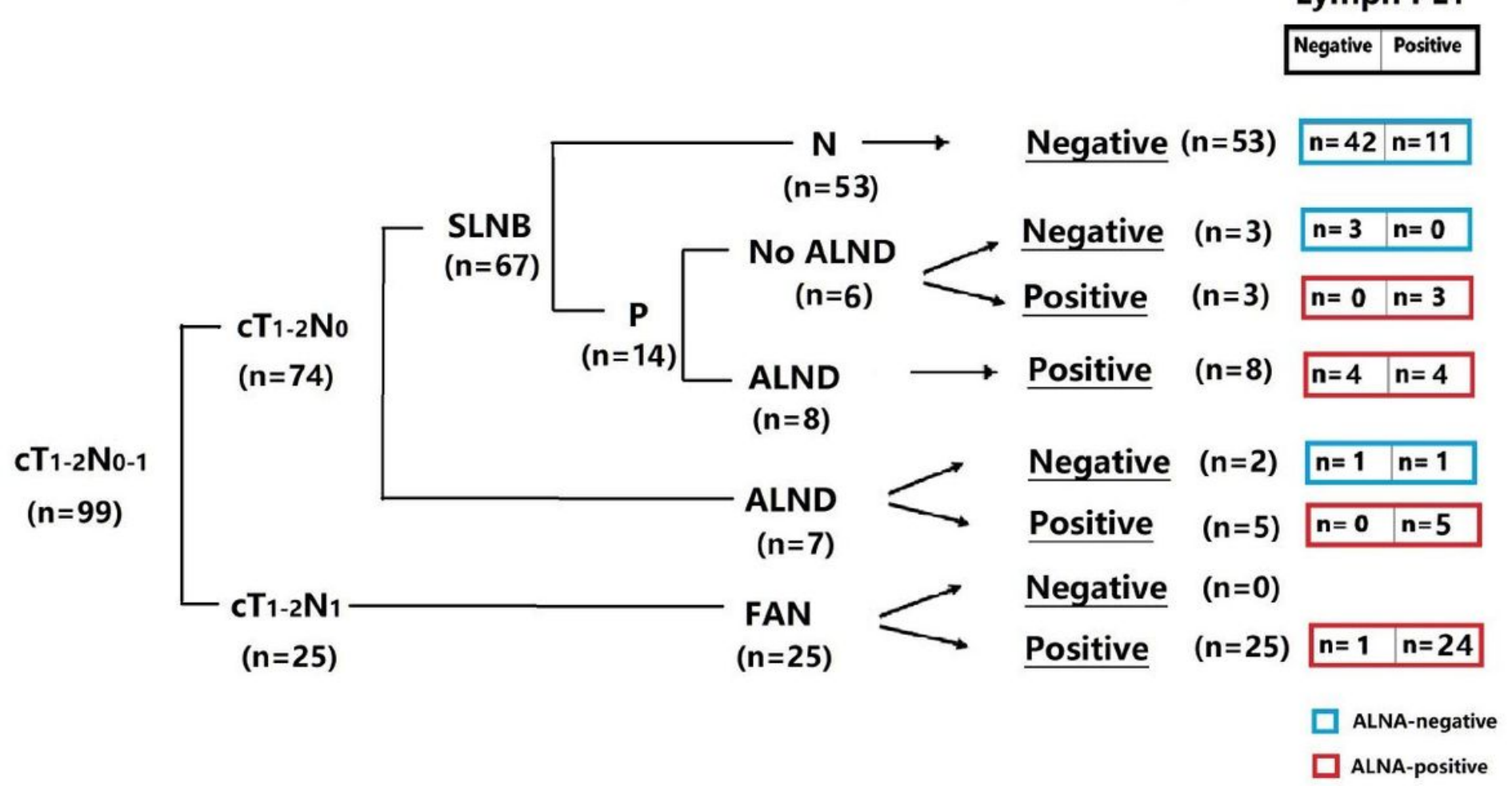

Figure 2

Consort diagram showing the results of axillary lymph node assessment (ALNA) and dedicated axillary positron emission tomography (LymphPET) scan. SLNB: sentinel lymph node biopsy; ALND: axillary nodal dissection. 
Figure 3.

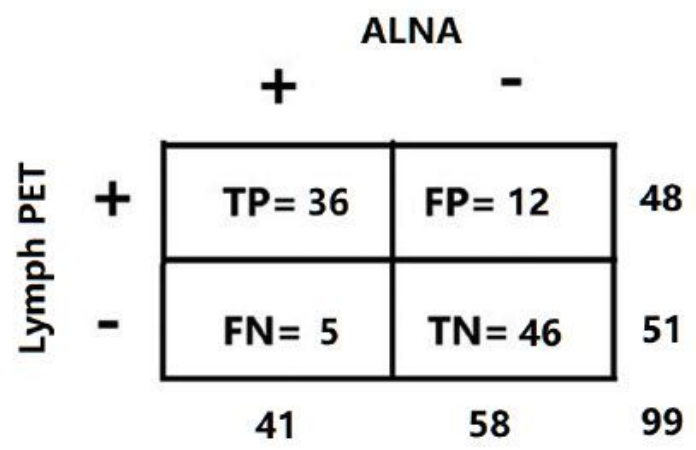

sensitivity $=87.80 \%$

specificity $=79.31 \%$

false-negative rate $=12.20 \%$

false-positive rate $=20.69 \%$

positive predictive value $(\mathrm{PPV})=75.00 \%$

negative predictive value (NPV) $=90.20 \%$

accuracy $=82.83 \%$

Figure 3

Estimates of diagnostic accuracy. ALNA: axillary lymph node assessment; TP: true-positive; FP: falsepositive; TN: true-negative; FN: false-negative. 
Figure 4.

a

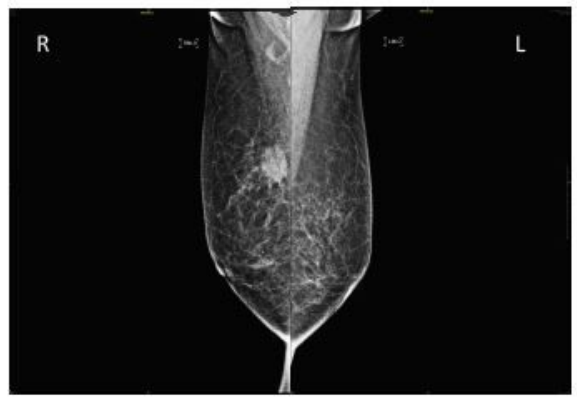

b
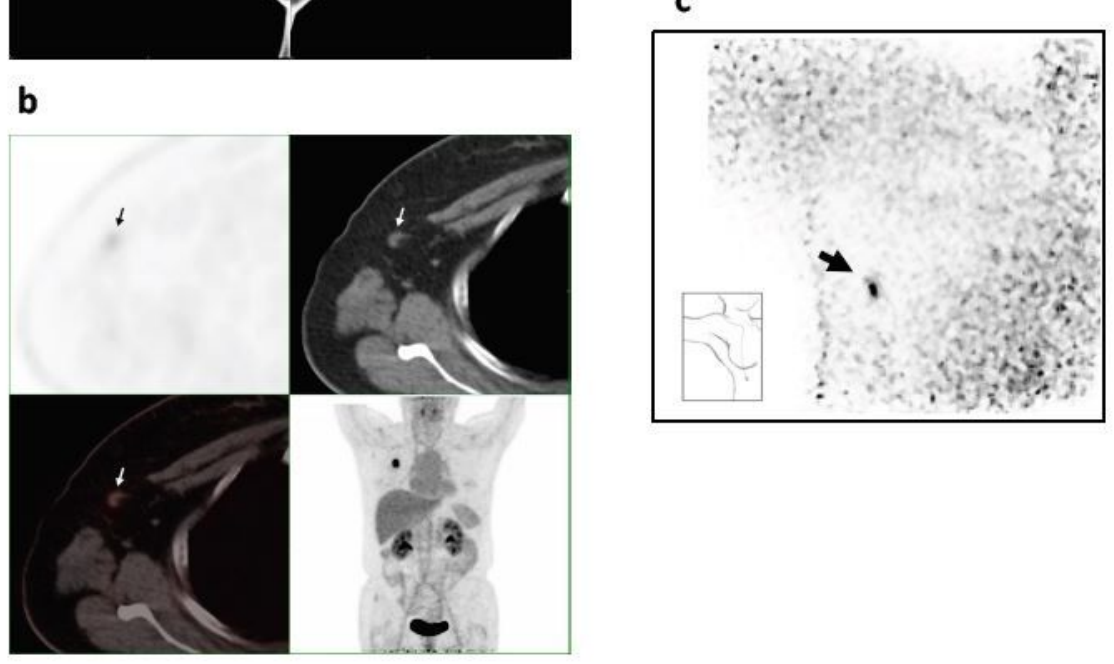

\section{Figure 4}

Typical true positive case. 57 years' old, right breast cancer (TNBC) without treatment ER(-), PR(-) ,HER2(0), ki67=40\% . US: Right axillary penetration and low echo, $12 * 7 \mathrm{~mm}$, regular shape, smooth edges, the lymphatic hilum structure is visible, and the cortex is thickened. Diagnosis: substantial lymph node in right axillary. a. Mammography before surgery: right breast showing a clear mass without ipsilateral axillary LN positive evident. b. Whole body PET/CT. A small Lymph Node in right axilla (arrow head) with 9*4mm sized and a slight FDG uptake with SUVmax=1.8 (<2.5). c. LymphyPET image. Right axillary Lymph Node (arrow head) with maxLUV=1.2 $(>0.27)$ and $7 * 4 \mathrm{~mm}$ size. Pathological diagnose: Right ALN metastasis. Surgery $\mathbb{A L N N D} 3 / 22(+)$ : three positive Lymph Nodes. 
Figure 5.

\section{a}

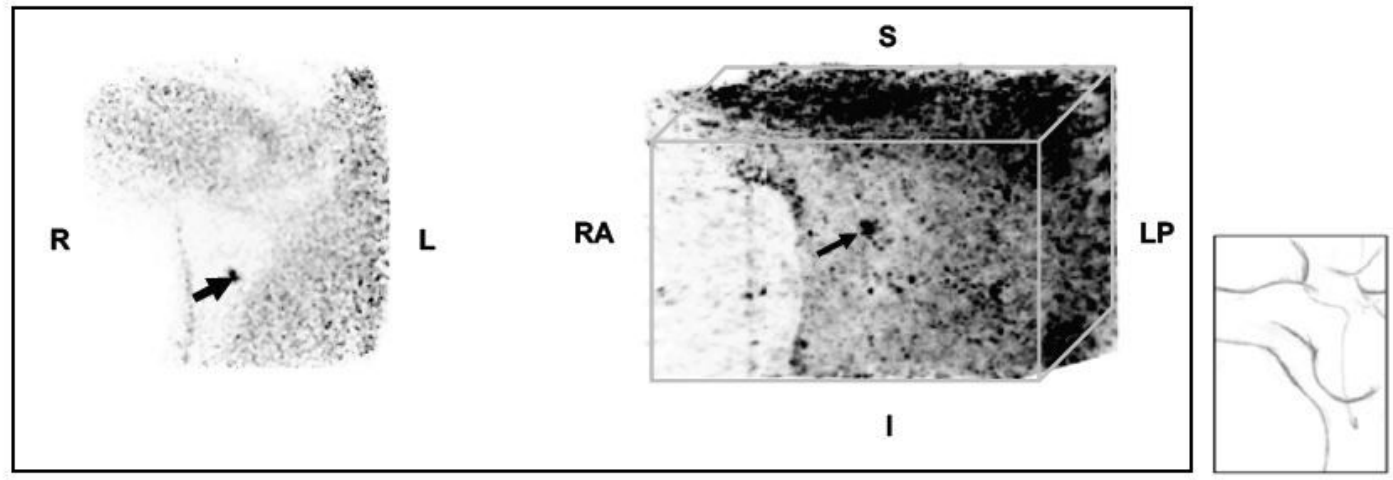

\section{b}

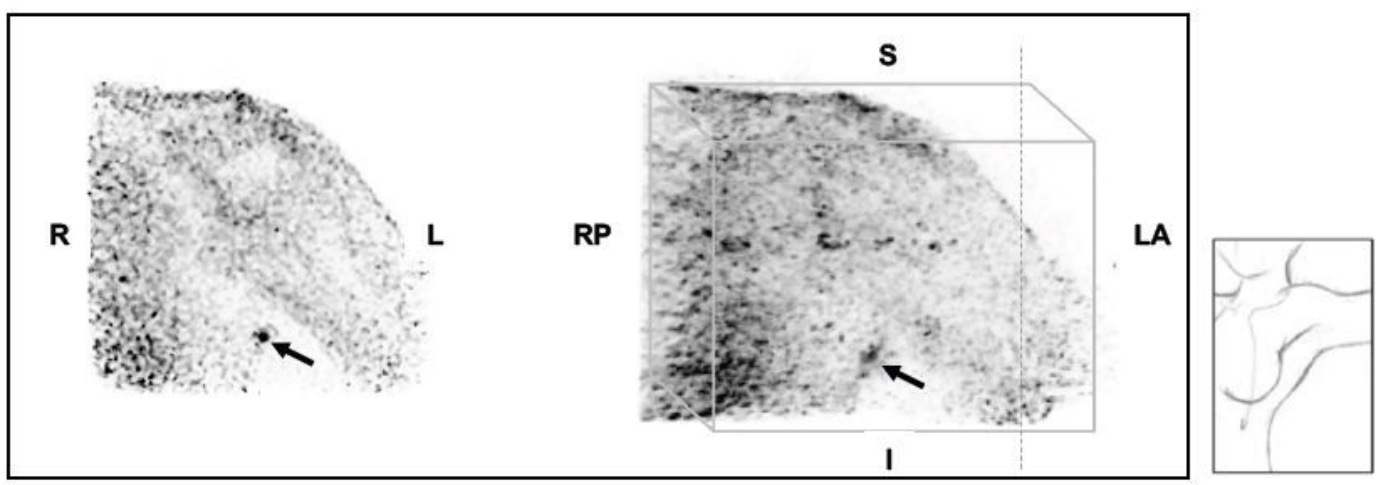

\section{Figure 5}

Examples of True Positive and False Positive with LymphPET. a. True positive. Forty-five-year-old woman with right invasive breast cancer of CT1NOMO. LymphPET images demonstrated a $7 \times 5 \mathrm{~mm}$ axillary node with focused fluorodeoxyglucose (FDG) uptake and the maximum single-voxel standard uptake value of axillary lymph node (maxLUV) was 1.2 (arrow), considered to be a metastasis. The patient underwent sentinel lymph node biopsy (SLNB) revealing two macro-metastases (2/5), and axillary lymph node dissection (ALND) showing no other metastasis (0/20). Single projection (left) and MIP (right) are shown. b. False positive. Forty-four-year-old woman with left invasive breast cancer of cT1N0M0. She could not raise her left arm because of scapulohumeral periarthritis. LymphPET shown a $6 \times 6 \mathrm{~mm}$ node with maxLUV=0.9. Image hot node located near the skin. SLNB showing no lymph node metastasis $(0 / 5)$ and the hot node was proved to be a pigmented nevus protruding from the surface of the skin. 
Figure 6.

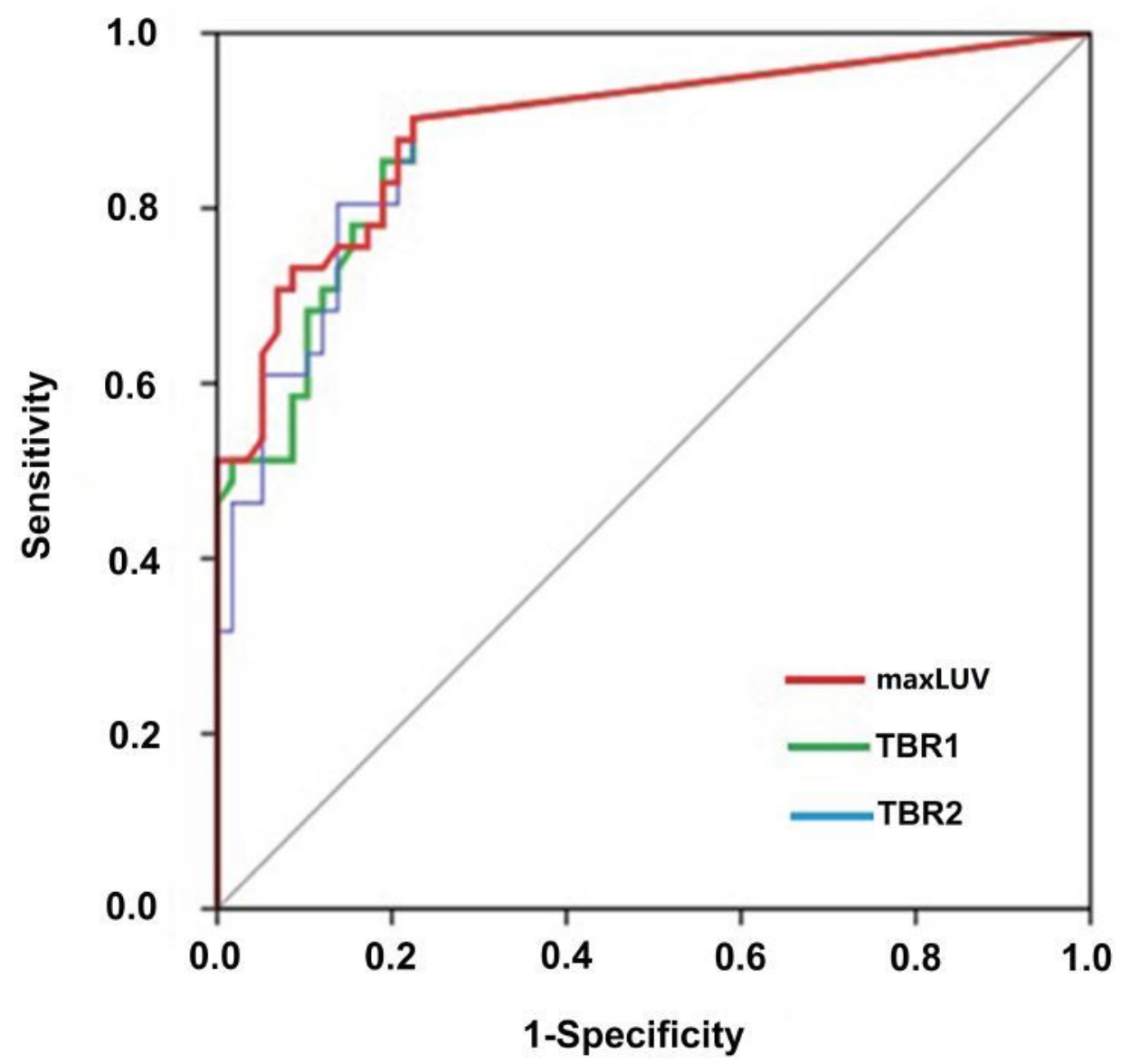

Figure 6

ROC curves of the three selected parameters for predicting nodal metastasis. AUC values for maxLUV, TBR1 and TBR2 were $0.895,0.885$ and 0.884 respectively. 